\title{
Andre Luiz Roizman
}

\section{Desenvolvimento de produtos para a} indústria de cartão de crédito: uma aplicação de análise conjunta

\author{
DISSERTAÇÃO DE MESTRADO
}

\section{DEPARTAMENTO DE ADMINISTRAÇÃO \\ Programa de Pós-Graduação em Administração de Empresas}




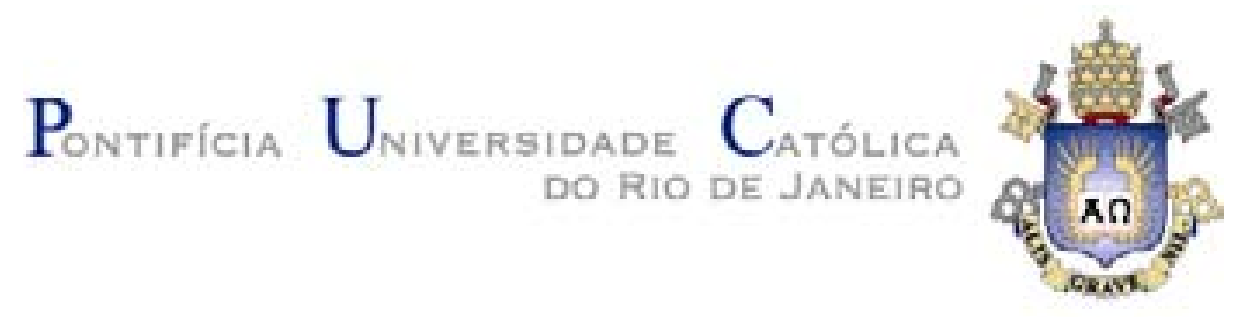

Andre Luiz Roizman

Desenvolvimento de produtos para a indústria de cartão de crédito: uma aplicação de análise conjunta

Dissertação de Mestrado

Dissertação apresentada como requisito parcial para obtenção do grau de Mestre pelo Programa de Pósgraduação em Administração de Empresas do Departamento de Administração da PUC-Rio.

Orientador: Prof. Jorge Ferreira da Silva

Rio de Janeiro

Outubro de 2003 


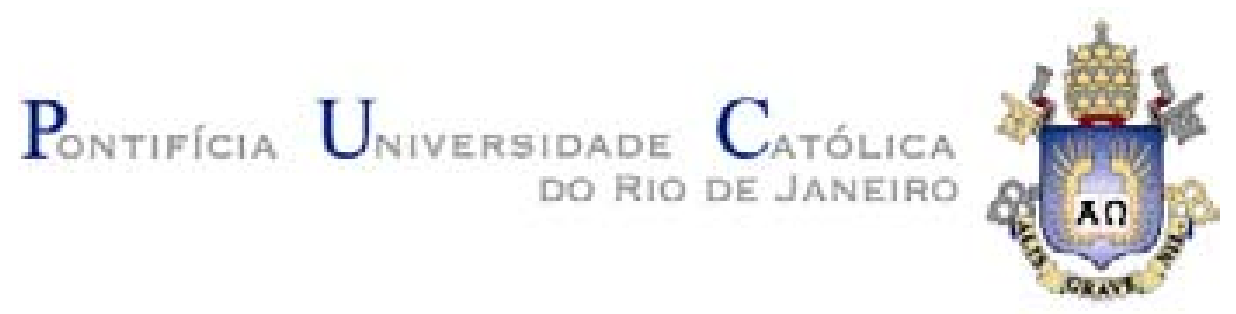

Andre Luiz Roizman

Desenvolvimento de produtos para a indústria de cartão de crédito: uma aplicação de análise conjunta

Dissertação apresentada como requisito parcial para obtenção do grau de Mestre pelo Programa de PósGraduação em Administração de Empresas do Departamento de Administração do Centro de Ciências Sociais da PUC-Rio. Aprovada pela Comissão Examinadora abaixo assinada

Prof. Jorge Ferreira da Silva
Orientador
Departamento de Administração, PUC-Rio

Prof. Paulo Cesar Motta

Departamento de Administração, PUC-Rio

Profa. Ângela Maria Cavalcanti da Rocha

COPPEAD, UFRJ

Profa. Zélia M. de Lossio e Seiblitz Coordenadora Setorial do Centro de Ciências

Sociais da PUC-Rio

Rio de Janeiro, 08 de Julho de 2003 
Todos os direitos reservados. É proibida a reprodução total ou parcial do trabalho sem autorização da universidade, do autor e do orientador.

\section{Andre Luiz Roizman}

Graduou-se em Administração de Empresas, na Universidade do Estado do Rio de Janeiro em 1995. Além do mestrado, possui MBA em Administração pelo Instituto Brasileiro de Mercados de Capitais, concluído em 2000. No âmbito profissional, atuou em diversas áreas, nas seguintes empresas: White Martins, Sistema PH de Ensino, Banco Bozano, Simonsen, Embratel e Light.

Ficha Catalográfica

\section{Roizman, Andre Luiz}

Desenvolvimento de produtos para a indústria de cartão de crédito : uma aplicação de análise conjunta / Andre Luiz Roizman; orientador: Jorge Ferreira da Silva. Rio de Janeiro : PUC, Departamento de Administração, 2003.

[16], 165 f. : il. ; $30 \mathrm{~cm}$

Dissertação (mestrado) - Pontifícia Universidade Católica do Rio de Janeiro, Departamento de Administração.

Inclui referências bibliográficas.

1. Administração - Teses. 2. Desenvolvimento de produtos. 3. Segmentação de mercado. 4. Marketing de serviços. 5. Análise conjunta. 6. Cartões de crédito. 7. Necessidades e desejos dos consumidores. I. Silva, Jorge Ferreira da. II. Pontifícia Universidade Católica do Rio de Janeiro. Departamento de Administração. III. Título.

CDD: 658 
Este trabalho é dedicado a:

Meus pais, irmão e a todos aqueles que contribuíram para a sua realização, com sugestões, paciência, apoio e incentivo nos momentos mais árduos, sem os quais o caminho até a sua conclusão teria sido muito mais difícil; 


\section{Agradecimentos}

Ao meu orientador, Professor Jorge Ferreira da Silva, pelo estímulo e ajuda para a realização deste trabalho.

Aos funcionários do IAG, pela disposição em ajudar-me a todo o momento, em especial a Teresa e ao Leopoldo.

Aos colegas da PUC-Rio, pelo incentivo e colaboração.

A todos os professores do Departamento, que de alguma forma me transmitiram ensinamentos valiosos.

A todos os colaboradores, que participaram do processo de definição de atributos e pesquisa de campo, envolvendo entrevistas, grupo de foco, pré-teste e respostas dos questionários.

Aos meus pais Hélio Henrique Roizman e Sonia Schneider Roizman e ao meu irmão Eduardo Roizman, por terem me proporcionado a educação que me levou a atingir os meus objetivos e pelo apoio e compreensão nestes anos de trabalho constante.

A minha noiva Débora Pires Agostinho da Silva pela paciência e incentivo nos últimos meses. 


\section{Resumo}

Roizman, Andre Luiz; Silva, Jorge Ferreira (Orientador). Desenvolvimento de produtos para a indústria de cartão de crédito: uma aplicação de análise conjunta. Rio de Janeiro, 2003. 165p. Dissertação de Mestrado Departamento de Administração, Pontifícia Universidade Católica do Rio de Janeiro.

O desenvolvimento de produtos e a correta distribuição aos segmentos de mercado são fatores fundamentais no processo de elaboração da estratégia de marketing das empresas a fim de proporcionar uma vantagem competitiva. Para isso, é essencial o perfeito entendimento das necessidades e desejos dos consumidores. Nessa dissertação, aplica-se a teoria de análise conjunta, com o objetivo de apontar o conjunto de características consideradas desejáveis pelos consumidores para a aquisição de cartões de crédito. $\mathrm{O}$ desenvolvimento do trabalho é composto de duas etapas: escolha dos atributos a serem avaliados pelos respondentes e análise dessa avaliação. Para escolher o conjunto de atributos a serem julgados foram realizadas entrevistas em profundidade e grupo de foco. A formação desse conjunto de estímulos foi desenvolvida utilizando-se o software SPSS 11.0 versão Windows. Em seguida, realizou-se a coleta de dados por meio de questionários, os quais foram enviados por correio eletrônico, sendo que alguns foram entregues pessoalmente. Por fim, também com o auxílio do software SPSS 11.0 versão Windows, o estudo atingiu seu objetivo, identificando o conceito de produto considerado mais adequado, fruto da combinação de estímulos que gerou maior nível de utilidade.

\section{Palavras-chave}

Desenvolvimento de Produtos; Segmentação de Mercado; Marketing de Serviços; Análise Conjunta; Cartões de Crédito; Necessidades e Desejos dos Consumidores. 


\section{Abstract}

Roizman, Andre Luiz; Silva, Jorge Ferreira (Advisor). Product development to credit cards industry: a conjoint analysis application Rio de Janeiro, 2003. 165p. MSc. Dissertation - Departamento de Administração, Pontifícia Universidade Católica do Rio de Janeiro.

The new product development and correct market segmentation are fundamental to define marketing strategy in order to provide a competitive advantage. To do this, the perfect understanding of customer needs is essential, too. In this research, the conjoint analysis theory is used with the objective of showing the group of characteristics understood as desirable by consumers to buy credit cards. This work is composed of two main basic steps: the choice of the attributes to be evaluated by the respondents and the analysis of this evaluation. The group of stimuli to be evaluated was chosen based on in-depth interviews and one focus group. The group of stimuli was developed using SPSS 11.0 software for Windows. Data collection was implemented with questionnaires sent by email. Some of them were handled personally. In the last step, also using the SPSS 11.0 software for Windows, this study reached its objective, identifying the concept of product considered the most adequate, as a result of the stimuli combination, which generated the highest utility level.

\section{Keywords}

Product Development; Market Segmentation; Services Marketing; Conjoint Analysis; Credit Cards; Customer Needs. 


\section{Sumário}

1. INTRODUÇÃO 16

1.1. Contextualização 18

1.2. Problema 20

1.3. Objetivos 22

1.4. Relevância do Estudo 23

1.5. Delimitação do Estudo 25

2. REFERENCIAL TEÓRICO 26

2.1. Marketing de Serviços 26

2.1.1. Características dos Serviços $\quad 27$

2.1.2. Classificação dos Serviços 28

2.2. Desenvolvimento de Produtos 32

2.2.1. Elaboração do Estudo (Design do Produto) 40

2.2.2. Coleta de Dados 41

2.2.3. Avaliação das Alternativas de Produtos 43

2.2.4. Vantagens da Utilização de Análise Conjunta 44

2.2.5. Limitações da Utilização de Análise Conjunta 45

2.2.6. Método Adotado 45

2.3. Segmentação de Mercado 47

2.3.1. Conceito 47

2.3.2. Bases e Metodologias para Segmentação 49

2.3.3. Processo de Segmentação 52

2.4. Mercado de Cartões de Crédito 54

2.4.1. Histórico do Cartão de Crédito 54

2.4.2. Entendendo o Mercado de Cartões de Crédito 55

2.4.3. Forma de Operação do Produto 56

2.4.4. Tendências 62

2.4.4.1. Cartão com Chip 62

2.4.4.2. Cartão Virtual 64 
3. METODOLOGIA DE PESQUISA 65

3.1. Tipo de Pesquisa 65

3.2. Universo e Amostra 66

3.2.1. Perfil da Amostra 66

3.3. Coleta de Dados 71

3.4. Tratamento dos Dados 72

3.5. Limitações do Método 73

3.6. Seleção de Atributos e Níveis 74

3.6.1. Entrevistas em Profundidade $\quad 74$

3.6.2. Grupo de Foco 79

3.7. Seleção das Opções de Compra 89

3.8. Pesquisa de Campo 92

4. RESULTADOS 94

5. CONCLUSÃO 126

5.1. Resumo 126

5.2. Sugestão para Estudos Futuros 129

6. REFERÊNCIAS BIBLIOGRÁFICAS 130

7. ANEXOS 135

7.1. Glossário 135

7.2. Base de Dados Fornecida pelos Respondentes $\quad 146$

7.3. Outras Informações do Mercado de Cartões de Crédito 152

7.3.1. Benefícios Oferecidos 152

7.3.2. Política de Segurança 153

7.3.3. Indicadores de Mercado 154

7.3.3.1. Gastos na Utilização de Cartões de Crédito 154

7.3.3.2. Volume de Operações de Crédito 154

7.3.3.3. Distribuição por Ramo de Atividade 155

7.3.3.4. Evolução do Mercado Internacional 156

7.3.3.5. Comparativo do Mercado Internacional 156

7.3.3.6. Pontos de Venda 158 
7.3.3.7. Perfil do Consumidor de Cartão de Crédito no Brasil Plano Real (1994 / 1998) 


\section{Lista de Tabelas}

Tabela 1 - Distribuição dos Meios de Pagamento 23

Tabela 2 - Potencial do Mercado de Cartões de Crédito 24

Tabela 3 - Classificação dos Atributos 84

Tabela 4 - Retorno de Questionários 92

Tabela 5 - Freqüências das Ordenações por Opções de Compra 93

Tabela 6 - Cartão Ideal para o Total da Amostra 95

Tabela 7 - Resultados para o Total da Amostra 96

Tabela 8 - Cartão Ideal para portadores de 1 Cartão 100

Tabela 9 - Cartão Ideal para portadores de 2 Cartões 100

Tabela 10 - Cartão Ideal para portadores de 3 Cartões 101

Tabela 11 - Resultados para portadores de 1 Cartão 102

Tabela 12 - Resultados para portadores de 2 Cartões 102

Tabela 13 - Resultados para portadores de 3 Cartões 103

Tabela 14 - Cartão Ideal para Gasto Médio Mensal até R\$ $\begin{array}{ll}200,00 & 107\end{array}$

Tabela 15 - Cartão Ideal para Gasto Médio Mensal de R\$ $\begin{array}{ll}200,00 \text { a } \mathrm{R} \$ 500,00 & 107\end{array}$

Tabela 16 - Cartão Ideal para Gasto Médio Mensal de R\$ 500,00 a R\$1.000,00 108

Tabela 17 - Cartão Ideal para Gasto Médio Mensal de R\$ $1.000,00$ a $R \$ 2.000,00 \quad 108$

Tabela 18 - Resultados para Gasto Médio Mensal até R\$ 200,00

Tabela 19 - Resultados para Gasto Médio Mensal de R\$ 200,00 a $\mathrm{R} \$ 500,00$

Tabela 20 - Resultados para Gasto Médio Mensal de R\$ 500,00 a $\mathrm{R} \$ 1.000,00$

Tabela 21 - Resultados para Gasto Médio Mensal de R\$ $1.000,00$ a $R \$ 2.000,00$ 
Tabela 22 - Cartão Ideal para Renda Mensal de R\$1.000,00 a $\mathrm{R} \$ 2.000,00$

Tabela 23 - Cartão Ideal para Renda Mensal de R\$2.000,00 a $\mathrm{R} \$ 5.000,00$

Tabela 24 - Cartão Ideal para Renda Mensal de $R \$ 5.000,00$ a $R \$ 10.000,00$

Tabela 25 - Resultados para Renda Mensal de $R \$ 1.000,00$ a $R \$ 2.000,00$

Tabela 26 - Resultados para Renda Mensal de $R \$ 2.000,00$ a $\mathrm{R} \$ 5.000,00$

Tabela 27 - Resultados para Renda Mensal de R\$5.000,00 a $\mathrm{R} \$ 10.000,00$

Tabela 28 - Cartão Ideal para Sexo Feminino

Tabela 29 - Cartão Ideal para Sexo Masculino

Tabela 30 - Resultados para Sexo Feminino

Tabela 31 - Resultados para Sexo Masculino

Tabela 32 - Gastos na Utilização de Cartões de Crédito

Tabela 33 - Volume de Operações de Crédito

Tabela 34 - Evolução do Mercado Internacional 


\section{Lista de Figuras}

Figura 1 - Características dos Serviços $\quad 28$

Figura 2 - Sistema de Classificação de Serviços 29

Figura 3 - Processo de Desenvolvimento de Produtos ou Serviços 35

Figura 4 - Base de Segmentação para Compradores Individuais $\quad 50$

Figura 5 - Bases e Variáveis de Segmentação 51 


\section{Lista de Gráficos}

Gráfico 1 - Possui Cartão de Crédito 67

Gráfico 2 - Bandeira $\quad 67$

Gráfico 3 - Renda Mensal 68

Gráfico 4 - Faixa Etária 68

Gráfico 5 - Região de Moradia $\quad 69$

Gráfico 6 - Sexo 69

Gráfico 7 - Gasto Médio Mensal com Cartões 70

Gráfico 8 - Percentual de Gastos com Cartões sobre Renda $\begin{array}{ll}\text { Mensal } & 70\end{array}$

Gráfico 9 - Anuidade $\quad 86$

Gráfico 10 - Limite Máximo $\quad 87$

Gráfico 11 - Limite Mínimo $\quad 87$

Gráfico 12 - Utilidade Bandeira - Todos 97

Gráfico 13 - Utilidade Preço - Todos 98

Gráfico 14 - Utilidade Limite - Todos 98

Gráfico 15 - Utilidade Benefícios - Todos 98

Gráfico 16 - Importância Relativa dos Atributos - Todos 99

Gráfico 17 - Utilidade Bandeira - Cartões 105

Gráfico 18 - Utilidade Preço - Cartões 105

Gráfico 19 - Utilidade Limite - Cartões 105

Gráfico 20 - Utilidade Benefícios - Cartões 106

Gráfico 21 - Importância Relativa dos Atributos - Cartões 106

Gráfico 22 - Utilidade Bandeira - Gasto Médio Mensal 112

Gráfico 23 - Utilidade Preço - Gasto Médio Mensal 112

Gráfico 24 - Utilidade Limite - Gasto Médio Mensal 113

Gráfico 25 - Utilidade Benefícios - Gasto Médio Mensal 113

Gráfico 26 - Importância Relativa dos Atributos - Gasto Médio

Mensal

Gráfico 27 - Utilidade Bandeira - Renda Mensal 119

Gráfico 28 - Utilidade Preço - Renda Mensal 119

Gráfico 29 - Utilidade Limite - Renda Mensal 120 
Gráfico 30 - Utilidade Benefícios - Renda Mensal

Gráfico 31 - Importância Relativa dos Atributos - Renda

Mensal

Gráfico 32 - Utilidade Bandeira - Sexo

Gráfico 33 - Utilidade Preço - Sexo

Gráfico 34 - Utilidade Limite - Sexo

Gráfico 35 - Utilidade Benefícios - Sexo

Gráfico 36 - Importância Relativa dos Atributos - Sexo

Gráfico 37 - Distribuição por Ramo de Atividade

Gráfico 38 - Volume de Transações

Gráfico 39 - Número de Cartões

Gráfico 40 - Posse e Ativação

Gráfico 41 - Plano Real - Sexo

159

Gráfico 42 - Plano Real - Idade

159

Gráfico 43 - Plano Real - Renda

159

Gráfico 44 - Plano Real - Região 